\title{
On the search for the origin of the bulge effect in high power laser beam welding
}

Cite as: J. Laser Appl. 31, 022413 (2019); https://doi.org/10.2351/1.5096133

Submitted: 14 March 2019. Accepted: 14 March 2019. Published Online: 17 April 2019

Antoni Artinov (D), Nasim Bakir (D), Marcel Bachmann (D), Andrey Gumenyuk (D), Suck-Joo Na (D), and Michael Rethmeier (D)

\section{COLLECTIONS}

Paper published as part of the special topic on Proceedings of the International Congress of Applications of Lasers \& Electro-Optics (ICALEO<sup>\&\#174;</sup> 2018)

Note: This paper is part of the Special Collection: Proceedings of the International Congress of Applications of Lasers (B)

\& Electro-Optics (ICALEO 2018).

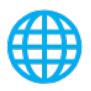

View Online

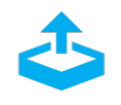

Export Citation

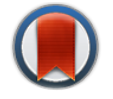

CrossMark

\section{ARTICLES YOU MAY BE INTERESTED IN}

Novel approach for weld depth determination using optical coherence tomography measurement in laser deep penetration welding of aluminum and steel

Journal of Laser Applications 31, 022007 (2019); https://doi.org/10.2351/1.5082263

Numerical analysis of the effect of welding positions on formation quality during laser welding of TC4 titanium alloy parts in aerospace industry

Journal of Laser Applications 31, 022401 (2019); https://doi.org/10.2351/1.5096095

Inline weld depth measurement for high brilliance laser beam sources using optical coherence tomography

Journal of Laser Applications 31, 022409 (2019); https://doi.org/10.2351/1.5096104

\section{Sellight Highlights of the best new research} in the physical sciences 


\title{
On the search for the origin of the bulge effect in high power laser beam welding
}

\author{
Cite as: J. Laser Appl. 31, 022413 (2019); doi: 10.2351/1.5096133 \\ Submitted: 14 March 2019 . Accepted: 14 March 2019. \\ Published Online: 17 April 2019
}

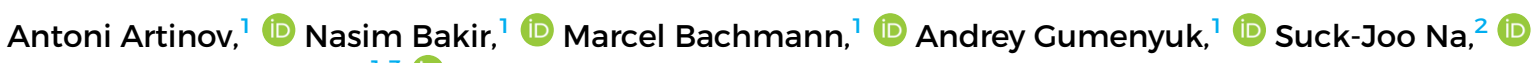
and Michael Rethmeier ${ }^{1,3}$ (D)

\begin{abstract}
AFFILIATIONS
${ }^{1}$ BAM Federal Institute for Materials Research and Testing, Unter den Eichen 87, Berlin 12205, Germany

${ }^{2}$ Department of Mechanical Engineering, KAIST, 291 Daehak-ro, Yuseong-gu, Daejeon 34141, South Korea

${ }^{3}$ Department of Joining Technology, Berlin Institute of Technology, 10623 Berlin, Germany
\end{abstract}

Note: This paper is part of the Special Collection: Proceedings of the International Congress of Applications of Lasers \& Electro-Optics (ICALEO ${ }^{\circledR}$ 2018).

\begin{abstract}
The shape of the weld pool in laser beam welding plays a major role in understanding the dynamics of the melt and its solidification behavior. The aim of the present work was its experimental and numerical investigation. To visualize the geometry of the melt pool in the longitudinal section, a butt joint configuration of $15 \mathrm{~mm}$ thick structural steel and transparent quartz glass was used. The weld pool shape was recorded by means of a high-speed video camera and two thermal imaging cameras, a mid-wavelength infrared camera and a newly developed infrared camera working in the spectral range of 500 to $540 \mathrm{~nm}$, making it perfectly suited for temperature measurements of molten materials. The observations show that the dimensions of the weld pool vary depending on the depth. The regions close to the surface form a teardrop-shaped weld pool. A bulge region and its temporal evolution were observed approximately in the middle of the depth of the weld pool. Additionally, a transient numerical simulation was performed until reaching a steady state to obtain the weld pool shape and to understand the formation mechanism of the observed bulging phenomena. A fixed keyhole with an experimentally obtained shape was used to represent the full-penetration laser beam welding process. The model considers the local temperature field, the effects of phase transition, thermocapillary convection, natural convection, and temperature-dependent material properties up to evaporation temperature. It was found that the Marangoni convection and the movement of the laser heat source are the dominant factors for the formation of the bulge region. A good correlation between the numerically calculated and the experimentally observed weld bead shapes and the time-temperature curves on the upper and bottom surface was found.
\end{abstract}

Key words: bulging effect, high power laser beam welding, process simulation, solidification, hot cracking

(C) 2019 Laser Institute of America. https://doi.org/10.2351/1.5096133

\section{INTRODUCTION}

In recent years, due to the significant development of laser sources and due to their unique advantages compared to conventional welding processes, laser beam welding of thick metal parts became an established processing tool allowing for increased industrial productivity. With the available high laser power of up to $100 \mathrm{~kW},{ }^{1,2}$ it becomes possible to weld samples of thickness up to $50 \mathrm{~mm}$ in a single pass welding. As a result of the high degree of focusability, especially when comparing the reachable radiation intensities to the local power deposition in multipass arc welding methods, such as Gas Metal Arc Welding and Submerged Arc Welding, the welding speed, penetration, and efficiency increase dramatically. Additionally, the net heat input needed to weld comparable samples in the case of laser beam welding is lower than that required by using the traditional processes. This provides the benefits of a narrow heat affected zone and minimizes the thermally induced welding distortion. ${ }^{3}$ Moreover, when using conventional welding methods, mostly multiple passes to meet the desired welding depth are inevitable, e.g., for the production of thick-walled pipelines for the oil and gas industry. ${ }^{4,5}$ Nevertheless, the laser beam welding technology associated with weld pool geometries of high depth-to-width ratio and different weighting of the underlying physics compared to traditional welding techniques requires a proper treatment and understanding of the underlying effects to avoid welding defects like 
solidification cracking, which are characteristic for this technique. Additionally, the complicated physics behind the laser beam welding process and the number of occurring physical phenomena make an estimation of the most important process quantities such as the temperature profile and the shape of the weld pool very challenging. The same also holds for the appropriate choice of the relevant process parameters like welding speed, laser power, and focal position.

Therefore, the aim of the present work was the experimental and the numerical investigation of the resulting weld pool shape obtained by high power laser beam welding with emphasis on the temporal and spatial evolution of the solidification region in the melt.

The occurring phenomena in the weld pool shape were identified being potentially beneficial for the formation of defects during the solidification stage, e.g., the hot cracking. Shida et al. have found a relation between the weld pool shape and the solidification cracking as they studied the effect of the welding parameters of electron beam welding on the weld quality. However, they have observed a small cavity in the rear part of the weld pool resulting through the variation of the focus position by the electron beam welding process. The observed cavity has shown significant influence on the solidification crack initiation. ${ }^{6}$ The locally delayed solidification phenomenon in the electron beam welding was also investigated from Tsukamoto et al. ${ }^{7}$ A close link between the delay in the solidification, the solidification cracking, and the porosity was observed. Gebhardt et al. ${ }^{8}$ have noted a similar phenomenon during laser beam welding of thick structures. They called it bulging in the weld. Their numerical models attributed a significant influence of the investigated bulging of the temperature field to the mechanical stress distribution, which is subsequently contributing to the formation of hot cracking during the solidification in the mushy zone (see Ref. 8). Barbetta et al. ${ }^{9}$ confirmed a strong relation between the bulging in the weld and the occurrence of solidification cracks, as the solidification crack was always associated with a bulge and thus a modification of the temperature distribution. Consequently, these phenomena, bulging and hot cracking, are related to each other by the delayed solidification at the rear part of the weld pool (see Ref. 9). To the best of the authors' knowledge, there is only little research on the occurrence of bulging during high penetration welding with lasers, although similar effects could be observed in recent numerical investigations (see Refs. 10-13).

In general, the weld pool shape is mainly affected by the dynamic interaction of the heat transport, fluid flow, and the liquid metal solidification behavior. To allow for a distinctive investigation of the elucidated bulging near the solidification front in high power laser beam welding, a butt configuration of transparent quartz glass and a low-alloyed steel was used in the present study. This configuration facilitates an optical observation and visualization of the weld pool shape in the longitudinal section and thus a qualitative deduction of the underlying effects during bulging as well as a comparison to numerical modeling.

A similar configuration of experiments was already performed by Li et al. ${ }^{14}$ to study the plasma and the keyhole behavior during high power deep penetration fiber laser beam welding of stainless steel. Zhang et al. ${ }^{15}$ also successfully used the butt configuration of quartz glass and zinc to study its behavior during laser beam welding. Wu et al. employed this experimental setup in combination with an external laser illumination to study the spatter formation in fiber laser welding of aluminum alloy. ${ }^{16}$
The main objective of the presented study was to analyses the weld pool shape in the longitudinal section qualitatively depending on the laser beam process in order to investigate the delay in the solidification front called bulging. Along with the numerical work presented in this paper, a high-speed video camera and two thermal imaging cameras, a mid-wavelength infrared (MWIR) camera and a newly developed infrared camera working in the spectral range of 500 to $540 \mathrm{~nm}$, making it perfectly suited for temperature measurements of molten materials, were employed to achieve this purpose on experimental basis.

\section{NUMERICAL MODELING}

It is a well-known fact that the physics representing the laser material interaction and thus the physics behind the laser beam welding process is very complex and highly coupled, which lead to difficulties in the numerical computation. Besides this fact, the proper numerical assessment of the shape of the weld pool and the thermal history of the welded part remains one of the most important tasks for engineers and researchers. In this paper, a methodology for the numerical solution of the Computational Fluid Dynamics (CFD) problem in the full-penetration keyhole laser beam welding process is presented. The numerical setup including the geometry of the workpiece, the initial state, and the boundary conditions can be seen in Fig. 1. The numerical model was implemented and solved with the commercial software ANSYS Fluent. The simulation of the process under investigation was done to obtain the quasi-steady-state weld pool geometry by computing the transient development of the weld pool geometry until reaching an equilibrium. It considers the most important physical phenomena decisive for the distributions of the velocity and the temperature field, namely, the effects of Marangoni convection, phase transition, natural convection, and temperaturedependent material properties up to evaporation temperature.

Since strongly coupled physical aspects and highly nonlinear system of equations are needed to be taken into account for the modeling of the laser beam welding process, a simplified form of the mathematical model was used to improve the numerical stability and to reduce the computing time. The basic assumptions made for the fluid flow and the temperature field simulation are similar

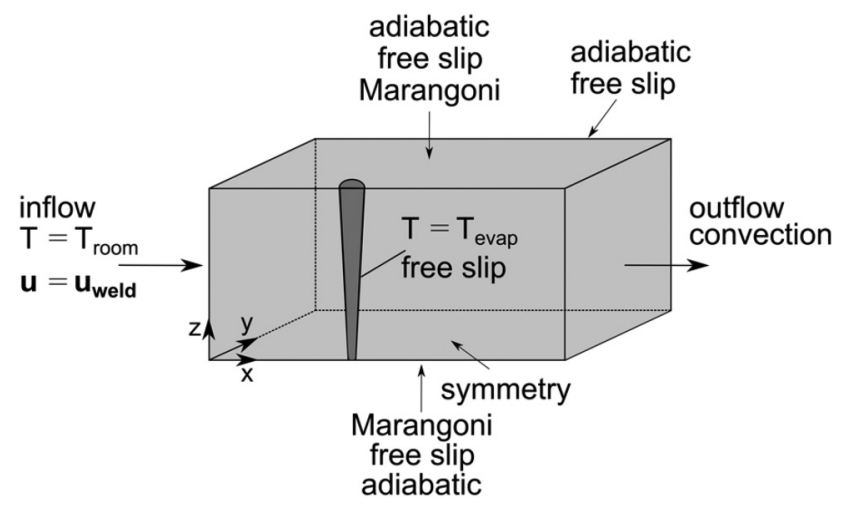

FIG. 1. Geometry, initial, and boundary conditions of the CFD model. 
to those used by Bachmann et al. in Refs. 17 and 18. They are as follows:

- The weld pool shape and the local temperature field were described by an Eulerian approach. Thus, effects caused by keyhole oscillations or unstable free surfaces were not considered.

- The size of the computational model was adapted to fulfill the adiabatic boundary conditions (see Fig. 1).

- The geometry of the free surfaces and the keyhole was fixed. Furthermore, an inclination of the keyhole geometry was not considered as the welding speed of the process was low. All dynamic effects of the recoil pressure on the melt pool were neglected and ideally balanced by surface tension forces.

- The keyhole geometry was simplified, and it was used as a model parameter to adapt the numerical to the experimental results (see Fig. 3).

- Shear stress due to the interaction of metal vapor and liquid metal was neglected.

- The amount of latent heat was considered by the apparent heat capacity method. ${ }^{19}$

- A turbulent flow pattern, based on the high velocities on both upper and lower sides, caused by the Marangoni driven flow, and the influence of the keyhole geometry on the flow, was considered by the standard $\kappa-\varepsilon$ turbulence model.

- Heat losses by radiation were not considered due to the high relation of volume versus surface of the plate.

- The basic equations representing the conservation of mass, momentum, and energy for incompressible flow were used, as they are implemented within the simulation framework of ANSYS Fluent to obtain the velocity and pressure fields. ${ }^{20}$ These were strongly coupled, whereby the coupling variables for the heat and mass transfer were the mass density, $\rho$, and the velocity vector of the molten metal, $\mathbf{u} .{ }^{20}$ Hence, the influence of the temperature distribution on the mass transport and vice versa was considered. In Fig. 2, the complex interaction of the single physics can be seen. The differential equations were adjusted to account for natural and thermocapillary convection, latent heat, and solidliquid phase transformation. The energy input was considered by setting the keyhole surface to the evaporation temperature of the material. The buoyancy force driven by the density deviation caused by the temperature difference was modeled by the wellknown Boussinesq approximation. ${ }^{21}$ The zone of the solid-liquid

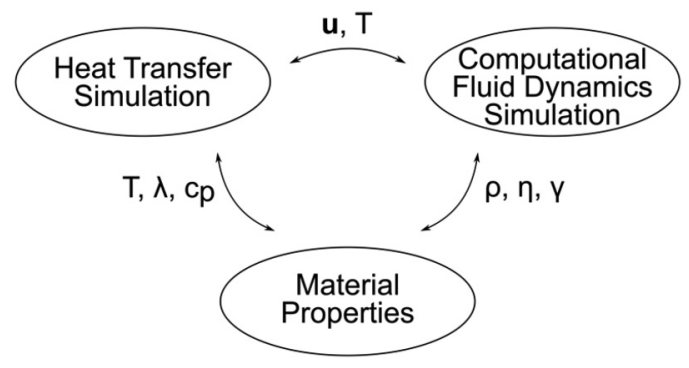

FIG. 2. Coupling variables used in the CFD simulation of the laser beam welding process.

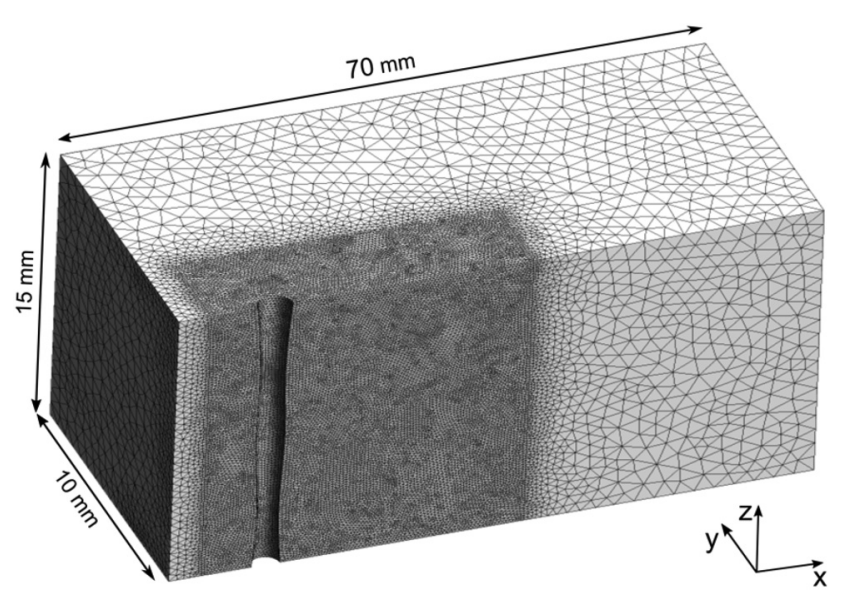

FIG. 3. Mesh used for the calculation.

phase transformation or also known as the "mushy zone" was considered by the Carman-Kozeny equation. This enthalpy-porosity approach allows the modeling of the welding process as a single phase by using a temperature-dependent liquid fraction, which calculates the amount of liquid in any element to be able to distinguish between the solid and the liquid phase. ${ }^{22}$ The needed model constants for the turbulence model were determined from experimental data. ${ }^{23}$ In addition, the Kays-Crawford heat transport model was used to account for the amount of turbulenceinduced heat conductivity. ${ }^{24}$ Thereby, the amount of fusion heat was released uniformly within the solid-liquid transformation zone. Hereby, the phase change temperature range in the model was chosen to be within a bandwidth of $50 \mathrm{~K}$. The geometrical dimensions of the model were $70 \times 10 \times 15 \mathrm{~mm}^{3}$ (see Fig. 3). A symmetry plane was introduced to the model; thus, the computational effort and time were significantly improved. The computational domain was discretized by a polygonal mesh of tetrahedral and triangular elements. The total number of mesh elements was about $1.1 \times 10^{6}$ and had a minimum element size of $0.1 \mathrm{~mm}$ at the free surfaces and the keyhole wall. The mesh is visualized in Fig. 3.

\section{EXPERIMENTAL SETUP}

For the welding experiments, a specially designed setup was necessary due to the optical inaccessibility to allow for the observation of the weld pool shape during high power laser beam welding. Therefore, butt welding experiments of $15 \mathrm{~mm}$ thick structural steel (S355) using a transparent quartz glass were conducted. The geometric dimensions of the workpiece are shown in the sketch of the experimental setup in Fig. 4. The laser beam welding process was performed with a fiber laser at a welding speed of $2 \mathrm{~m} / \mathrm{min}$, a laser power of $18 \mathrm{~kW}$, a focal position of $-5 \mathrm{~mm}$, and a focal diameter of $0.56 \mathrm{~mm}$.

The transparent quartz glass used in the present investigation was chosen due to its adequate physical properties. The material 


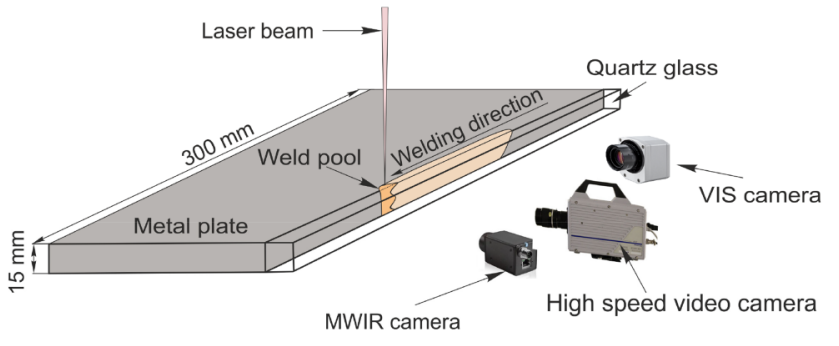

FIG. 4. Sketch of the experimental setup for butt joint configuration of structural steel S355 and quartz glass.

offers a suitable transparency for the laser wavelength of $1070 \mathrm{~nm}$ - the transmission properties for wavelengths between $200 \mathrm{~nm}$ and $2500 \mathrm{~nm}$ were up to $90 \%$. Due to its unique properties such as low thermal conductivity, low thermal expansion coefficient, excellent thermal shock resistance, and the high melting temperature being all not comparable to those of the used steel, the glass-steel interface was considered as the plane of symmetry of the welding process. The center of the laser spot was adjusted to lie exactly in the symmetry plane thus distributing half of the laser radiation on the steel plate and the other half on the quartz glass. The geometric characteristics of the resulting weld pool in the symmetry plane between the quartz glass and the metal plate were observed using an infrared camera, a thermo camera, and a high-speed camera from the glass side simultaneously. The sensor in the infrared camera allowed for a detection of an MWIR light, e.g., from 1000 $\mathrm{nm}$ up to $5000 \mathrm{~nm}$. This intersection between the spectral transmission of the glass and the detecting range of the infrared camera allowed the monitoring of the thermal profile of the weld pool through the glass. Additionally, the results of the operating in visual spectral range thermo camera were added to determine the shape of the solidification isotherm of the weld pool.

The used thermo camera works with the special wavelength range of $500-540 \mathrm{~nm}$. This unique measuring range neutralizes the laser radiation and their reflections. The quartz glass for this wavelength range is fully transparent, which allows the measurements without any hindering to the measuring wave.

The performed experiments were carried out aiming at a qualitative observation of the geometry of the weld pool in the symmetry plane.

\section{RESULTS AND DISCUSSION}

From the high-speed camera images, it was observed that the dimensions of the melt vary depending on the penetration depth with respect to time (see Fig. 5).

The content of the observed region was a mixture of liquid metal, metal vapor, and laser reflections on the keyhole surfaces. The bulging region monitored by the high-speed camera was located near but outside the keyhole region. The measurements of the infrared and the thermo camera were used to confirm the observations of the high-speed camera imaging (see Figs. 6 and 7). Hence, this bright region defined by the yellow line represented an

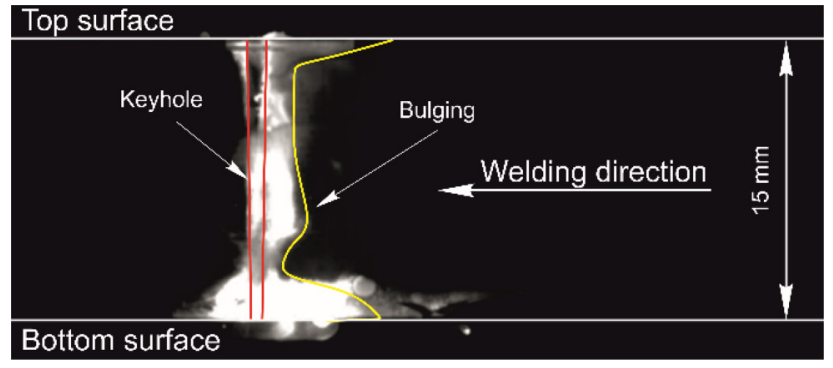

FIG. 5. View of the longitudinal section through the quartz glass using a high-speed camera.

isotherm within the zone of the molten metal, which is bounded by the isotherms of the evaporation and the solidus temperatures. Consequently, it was expected that the observed bulge in the mixture influenced the solidification front thus changing the weld pool geometry.

The longitudinal view of the weld pool in the symmetry plane showed a delay in the solidification behavior of the material in the middle of the symmetry plane. The delay and the related thereto hot crack formation in the bulging region are represented in Fig. 8(a). In Fig. 8(b), cross section with a longitudinal hot crack appearing in the bulging region is shown. The areas in the vicinity of the top and bottom surfaces of the weld pool were found to be teardrop-shaped. Two necking areas, situated between the bulge, the upper, and the lower parts of the weld pool were identified. In addition, the qualitative shape of the solidification front in the longitudinal direction was assumed to be a parallel shift of the shape of the bright region, defined by the yellow line in Fig. 5 .

However, the experimental results were not sufficient for a quantitative investigation of the weld pool shape. To improve the understanding of this problem by giving a physical explanation of the origin of the lower and upper necking as well as the bulging region numerical simulation was used. The numerically obtained results allowed to analyze the interaction of the different physical aspects. Thus, their impact on the weld pool geometry can be evaluated.

The aim of the proposed numerical model was to predict the size and the shape of the melt pool in the steady-state zone of the weld. Thus, the most important physical effects for the heat and mass transfer, such as thermocapillary convection, latent heat, and buoyancy force, were considered. The outer contour of the

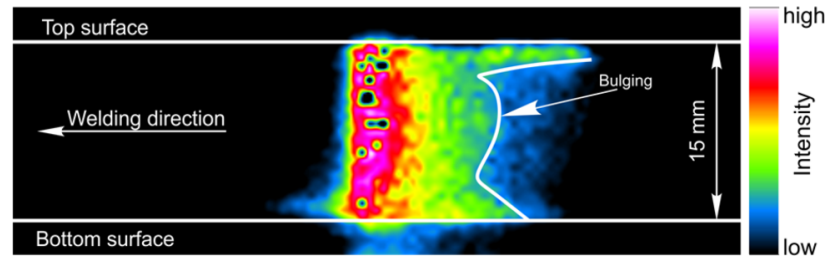

FIG. 6. View of the longitudinal section through the quartz glass using an infrared camera. 

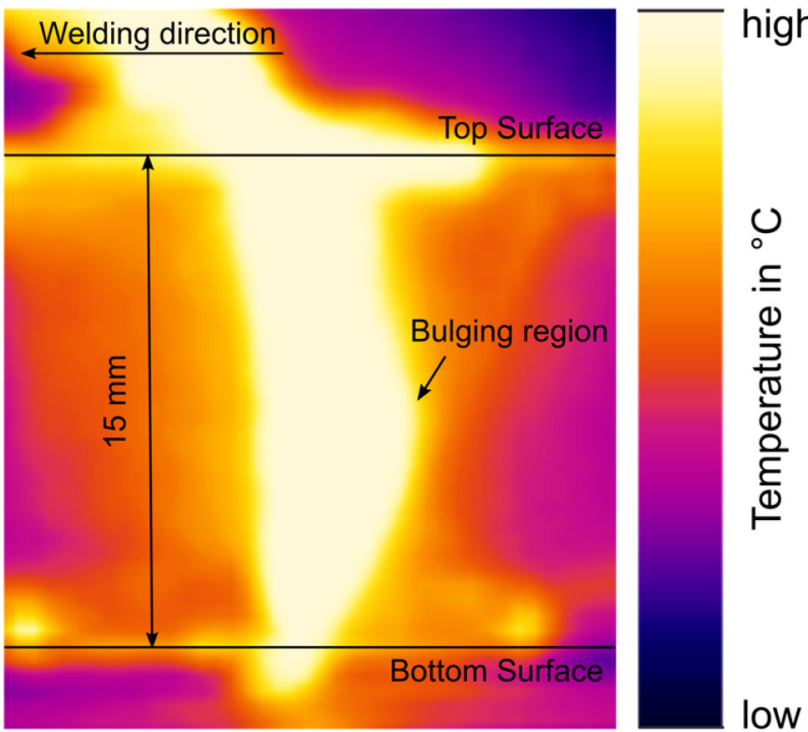

FIG. 7. View of the longitudinal section through the quartz glass using a thermo camera.

three-dimensional weld pool geometry is defined by the liquidus temperature and can be seen in Fig. 9.

The liquidus isotherm in the symmetry plane of the weld is also shown in Fig. 10. The final steady-state geometry of the weld pool can be classified in three regions. The regions on the upper and lower part of the specimen and the bulge region in the middle a)

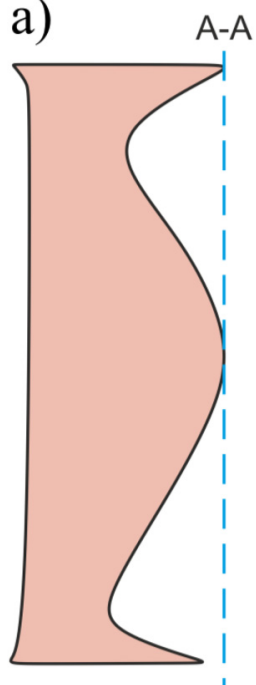

A-A

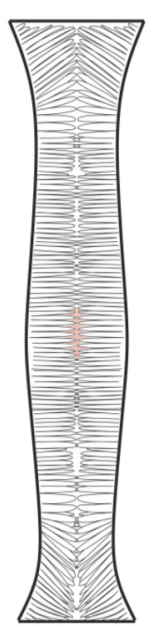

b)

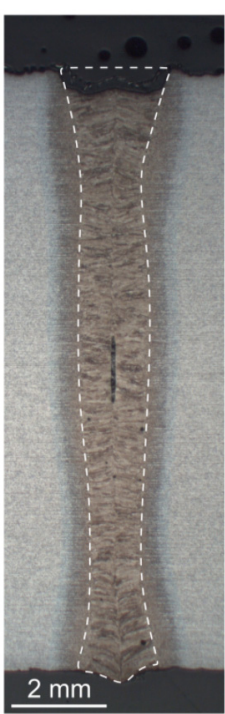

FIG. 8. (a) Schematic representation of the formation of a hot crack in the experimentally observed bulging region. (b) Cross section with a longitudinal hot crack appearing in the bulging region.

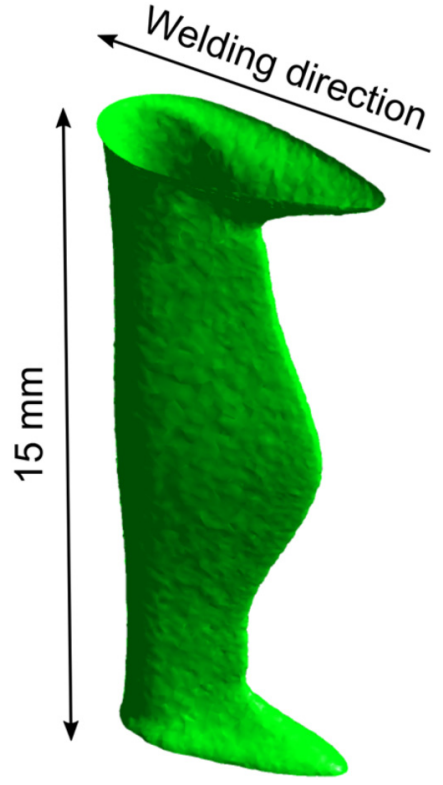

FIG. 9. Computed three-dimensional weld pool shape defined by the liquidus temperature.

of the plate. The liquid metal flow directions with the highest impact on the weld pool behavior were identified and are shown in Fig. 11. Their interaction determines the quasi-steady-state weld pool geometry, which travels through the specimen by means of the movement of the laser spot with respect to time. The simulation was used to improve the understating of the formation and the

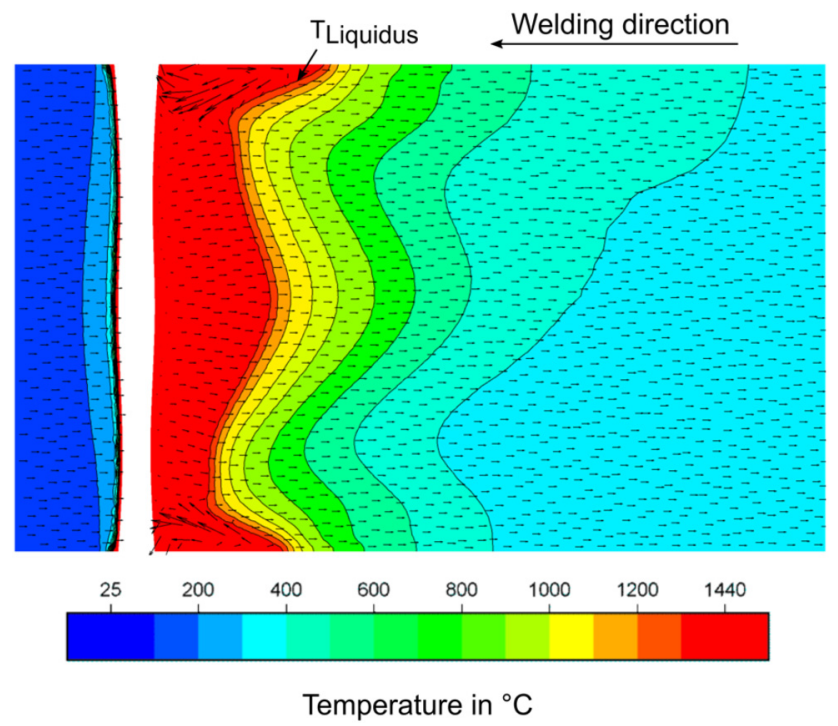

FIG. 10. Numerically obtained temperature and velocity fields in the symmetry plane of the weld. 


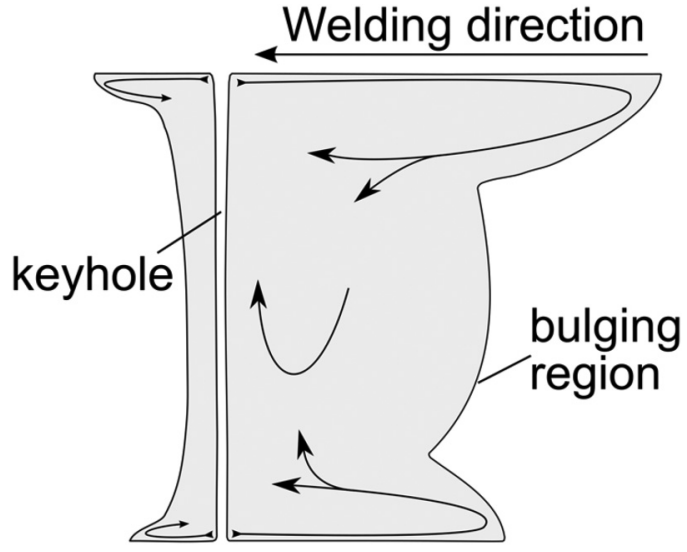

FIG. 11. Schematic view of the main flow directions in the weld pool in the symmetry plane of the numerical model.

development of the main flows in the weld pool. The decrease in the temperature values along the weld pool surface away from the keyhole results in an increase of the corresponding local surface tension. Due to this, the gradient of the surface tension increases and the melt is accelerated away from the keyhole by tangential shear stresses. As it can be seen, the regions on the upper and lower side in front of the keyhole caused by the Marangoni convection are very small in comparison to those behind the keyhole. That can be easily explained by the difference in the amount of molten material in the two areas. However, it must also be mentioned here that the isotherms in front of the keyhole lie much closer to each other, which results in a steeper temperature gradient and consequently causes a higher acceleration of the melt. The calculated velocity of the molten metal within the weld pool is very small compared to its maximum calculated at the free surfaces in front of the keyhole. The analysis of the numerical results showed that the influence of the natural convection on the weld bead geometry is very small in comparison to the Marangoni convection. Hence, this effect could be neglected in further computations. The shape of the middle region is believed to be mainly influenced by the experimentally observed bulge behind the keyhole and the high processing speed. The observed velocities of the molten material in the middle region were very small compared to the values in the upper and lower region. Hence, the edge of the middle region is nearly a parallel shift of the keyhole edge, which forms, together with the two backflows due to the Marangoni effect, the observed bulge. The weld pool in the steady-state zone of the laser beam welding process represents a simple thermal and mass equilibrium defined by the movement of the laser source and the solidification speed at the trailing part of the molten pool. By moving the laser head, the cold material in front of it is molten and added to the melt pool. This is then transferred by the different flows in the weld pool. In steady-state, the same amount of melt solidifies at the rear side of the weld. In the present model, the thermocapillary driven flow dominates, and a cooler material is driven back to the vicinity of the keyhole ensuring the mass conservation. Hence, the two necking areas and consequently the bulge are formed (see Fig 10).
By simply neglecting the local convection in the molten pool and considering the trivial case of heat transfer with a constant convective term in the flow equations, the rear edge of the weld pool would be a simple parallel shift to the keyhole boundary caused by the movement of the laser spot. Without the backflows on the upper and lower side due to Marangoni convection, there will be no bulge in the vertical center of the weld pool. Thus, the interaction of the physical effects, and here mainly due to the surface tension driven acceleration of melt at the free surfaces, plays a significant role in forming the spatial characteristics of the weld pool.

\section{CONCLUSIONS}

In this work, experimental and numerical analyses of the weld pool shape in laser welding of a low-alloyed steel (S355) were carried out.

Experimentally, a depth dependency of the weld pool dimensions was found. At the top and the bottom surfaces, the weld pool has taken a teardrop shape, whereas a bulging approximately in the middle of the depth was observed. The bulging was separated from the top and the bottom with necking areas.

In the numerical simulation, a simplified model was developed to reproduce the experimentally observed phenomena and to improve the understanding of the formation and the development of the bulging. However, this model allowed to analyze and thus to understand more deeply the formation of the bulging mechanism in the laser beam welding process. According to the simulation results, it can be concluded that the interaction of the movement of the laser source with the Marangoni vortex leads to a teardrop shape at the upper and bottom surface of the workpiece. Additionally, it shows that the bulging in the weld is a result of the backflows on the upper and lower side due to the thermocapillary driven flows.

Definitely, the observed weld pool shape has a significant influence on the time-related and local stress distribution during solidification and consequently on the solidification cracking, which needs further investigations.

\section{ACKNOWLEDGMENTS}

Financial funding of the Deutsche Forschungsgemeinschaft (DFG, German Research Foundation) under Grant No. BA 5555/1-1 is gratefully acknowledged. This work was supported by the Research Association for Steel Application (FOSTA), the Federation of Industrial Research Associations (AiF), and the German Federal Ministry for Economic Affairs and Energy (BMWi Bundesministerium für Wirtschaft und Energie) (Project No. 19582N, 'Investigation of the influence of the restraint conditions on hot cracking in laser and laser-hybrid welding of thick structure steels').

\section{REFERENCES}

${ }^{\mathbf{1}}$ M. Bachmann, A. Gumenyuk, and M. Rethmeier, "Welding with high-power lasers: Trends and developments," Phys. Procedia 83, 15-25 (2016).

${ }^{2}$ X. Zhang, E. Ashida, S. Tarasawa, Y. Anma, M. Okada, S. Katayama, and M. Mitzutani, "Welding of thick stainless steel plates up to $50 \mathrm{~mm}$ with high brightness lasers," J. Laser Appl. 23, 022002 (2011).

${ }^{3}$ LIA Handbook of Laser Materials Processing, edited by J. F. Ready and D. F. Farson (Laser Institute of America, Orlando, FL, 2001). 
${ }^{4}$ S. Gook, A. Gumenyuk, and M. Rethmeier, "Orbital laser-hybrid welding of pipelines using a $20 \mathrm{~kW}$ fibre laser," in Proceedings of the 5th International WLT-Conference on Lasers in Manufacturing, Munich, 15-18 June 2009 (German Scientific Laser Society, WLT, Stuttgart, 2009), pp. 65-70

${ }^{\mathbf{5}}$ S. Gook, A. Gumenyuk, and M. Rethmeier, "Weld seam formation and mechanical properties of girth welds performed with laser-GMA-hybrid process on pipes of grade X65," in Proceedings 29th International Congress on Applications of Lasers and Electro-Optics, Anaheim, CA, 26-30 September 2010 (ICALEO, Anaheim, CA, 2010).

${ }^{6}$ T. Shida, H. Okumura, and Y. Kawada, "Effects of welding parameters and prevention of defects in deep penetration electron beam welding of heavy section steel plates," Weld. World 17, 196-207 (1979).

${ }^{7}$ S. Tsukamoto and H. Irie, "Mechanism of locally delayed solidification in electron beam welding," Weld. Int. 5, 177-183 (1991)

${ }^{8}$ M. Gebhardt, A. Gumenyuk, and M. Rethmeier, "Numerical analysis of hot cracking in laser-hybrid welded tubes," Adv. Mater. Sci. Eng. 2013, 1-8 (2013).

${ }^{9}$ L. D. Barbetta, W. L. Weingaertner, O. Seffer, R. Lahdo, and S. Kaierle, "Influence of molten pool geometry and process parameters on solidification cracks formation in hybrid laser-GMA welding of thick 5L X70 steel plates" in $\mathrm{ABCM}$ International Congress of Manufacturing Engineering (COBEM), 8th Brazilian Congress of Manufacturing Engineering, Salvador, Bahia, Brazil, 18-22 May 2015.

${ }^{10}$ W. I. Cho, S. J. Na, C. Thomy, and F. Vollertsen, "Numerical simulation of molten pool dynamics in high power disk laser welding," J. Mater. Proc. Technol. 212, 262-275 (2012).

${ }^{11}$ M. Sohail, S. W. Han, S. J. Na, A. Gumenyuk, and M. Rethmeier, "Numerical investigation of energy input characteristics for high-power fiber laser welding at different positions,” Int. J. Adv. Manuf. Technol. 80, 931-946 (2015).

${ }^{12}$ F. Lu, X. Li, Z. Li, X. Tang, and H. Cui, "Formation and influence mechanism of keyhole-induced porosity in deep-penetration laser welding based on 3D transient modelling," Int. J. Heat Mass Transf. 90, 1143-1152 (2015).

${ }^{13}$ Z. Gao, P. Jiang, G. Mi, L. Cao, and W. Liu, "Investigation on the weld bead profile transformation with the keyhole and molten pool dynamic behavior simulation in high power laser welding," Int. J. Heat Mass Transf. 116, 1304-1313 (2018).

${ }^{14} \mathrm{~S}$. Li, G. Chen, M. Zhang, Y. Zhou, and H. Deng, "Investigation of keyhole plasma during $10 \mathrm{~kW}$ high power fiber laser," Laser Phys. 24, 106003 (2014).

${ }^{15}$ Y. Zhang, S. Li, G. Chen, H. Zhang, and M. Zhang, "Characteristics of zinc behavior during laser welding of zinc "sandwich" sample," Opt. Laser Technol. 44, 2340-2346 (2012).

${ }^{16}$ D. Wu, X. Hua, Y. Ye, L. Huang, F. Li, and Y. Huang, "Experimental and numerical study of spatter formation and composition change in fiber laser welding of aluminum alloy," J. Phys. D Appl. Phys. 51, 185604 (2018).

${ }^{17}$ M. Bachmann, V. Avilov, A. Gumenyuk, and M. Rethmeier, "Numerical simulation of full-penetration laser beam welding of thick aluminium plates with inductive support," J. Phys. D Appl. Phys. 45, 035201 (2011).

${ }^{18}$ M. Bachmann, R. Kunze, V. Avilov, and M. Rethmeier, "Finite element modeling of an alternating current electromagnetic weld pool support in full penetration laser beam welding of thick duplex stainless steel plates," J. Laser Appl. 28, 022404 (2016).

${ }^{19}$ H. T. Hashemi and C. M. Sliepcevich, "A numerical method for solving twodimensional problems of heat conduction with change of phase," Chem. Eng. Prog. Symp. Series 63, 34-41 (1967).

${ }^{20}$ J. Larsson, "Numerical simulation of turbulent flows for turbine blade heat transfer applications," Ph.D. thesis, Chalmers University of Technology, 1998.

${ }^{21}$ T. E. Faber, Fluid Dynamics for Physicists (Cambridge University Press, Cambridge, 1995).

${ }^{22}$ A. D. Brent, V. R. Voller, and K. T. J. Reid, "Enthalpy-porosity technique for modeling convection-diffusion phase change: application to the melting of a pure metal," Num. Heat Transf. 13, 297-318 (1988).

${ }^{23}$ D. C. Wilcox, Turbulence Modeling for CFD (DCW Industries, La Cañada Flintridge, CA, 1998), Vol. 2, pp. 103-217.
${ }^{24}$ W. M. Kays, "Turbulent Prandtl number-Where are we?" J. Heat Transf. 116, 284-295 (1994).

\section{Meet the Authors}

Antoni Artinov, born in 1991 in Aytos (Bulgaria), accepted his M.Sc. in the field of Engineering Science from the Berlin Institute of Technology and his M.Sc. in Mechanical Engineering from the Tomsk Polytechnic University. Since 2016, he is working as a research assistant at the Federal Institute for Materials Research and Testing in Berlin, Germany, in the "Welding Technology" department. Currently, he is working in the field of numerical simulations in laser beam welding processes.

Nasim Bakir received his diploma from the Aleppo University in the faculty of Mechanical Engineering (material science department). After his study, he was a lecturer at the Aleppo University for the topic Finite Elements Method (2008-2010). Next, he was a guest scientist at the Berlin Institute of Technology in the Department of Surface and Joining Technology (2010-2013). Since 2013, he is a researcher at the Federal Institute for Materials Research and Testing (BAM) in Berlin. He focuses on laser beam welding, hybrid laser arc welding, simulation of beam welding processes, and hot cracking phenomena.

Dr.-Ing. Marcel Bachmann, born 1984 in Berlin, is with the BAM Federal Institute for Materials Research and Testing in Berlin, Germany, in the "Welding Technology" department since 2009 where he is leading the working group "Welding Simulation." $\mathrm{He}$ received his diploma from the Berlin Institute of Technology in Engineering Science and his Ph.D. for numerous investigations in electromagnetically assisted high power laser beam welding processes. Currently, he is working on several projects involving numerical simulations in welding processes.

Dr.-Ing. Andrey Gumenyuk is the head of the working group "Laser beam welding processes" at the BAM Federal Institute for Materials Research and Testing in Berlin, Germany, in the "Welding Technology" department. He received his Ph.D. from the Technical University (RWTH) Aachen in Mechanical Engineering. The focus of his work is high power laser and laser arc hybrid welding technologies.

Professor Na is working at KAIST, Korea, in the field of simulation and optimization of arc, laser, and laser arc hybrid welding process, in which he links arc physics and multiple reflections in keyhole with weld pool dynamics. Recently, he has commenced with the CFD simulation of FSW and SLM process, and also with the metallurgical and mechanical optimization of welded structures using advanced digital simulation technologies. He has published 176 research papers in international journals and presented more than 140 papers at international welding conferences.

Professor Dr.-Ing. Michael Rethmeier is with the BAM Federal Institute for Materials Research and Testing. He is the head of the division "Welding Technology." He is also heading the "Chair of Joining Technology" at the Institute of Machine Tools and Factory Management, Berlin Institute of Technology, and is division director of "Joining and Coating Technology" at the Fraunhofer Institute for Production Systems and Design Technology. Present research topics include among others innovative arc welding processes, high power laser beam welding, and numerical simulations in various welding processes. 